\title{
Improving quality of care and clinical outcomes for rectal cancer through clinical audits in a multicentre cancer care organisation
}

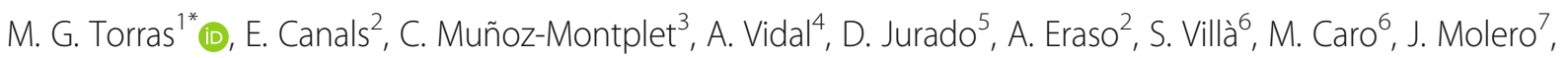 \\ M. Macià ${ }^{8}$, M. Puigdemont ${ }^{9}$, E. González-Muñoz ${ }^{4}$, A. López ${ }^{10}$, F. Guedea ${ }^{11}$ and J. M. Borras ${ }^{12}$
}

\begin{abstract}
Introduction: Colorectal cancer treatment requires a complex, multidisciplinary approach. Because of the potential variability, monitoring through clinical audits is advisable. This study assesses the effects of a quality improvement action plan in patients with locally advanced rectal cancer and treated with radiotherapy.

Methods: Comparative, multicentre study in two cohorts of 120 patients each, selected randomly from patients diagnosed with rectal cancer who had initiated radiotherapy with a curative intent. Based on the results from a baseline clinical audit in 2013, a quality improvement action plan was designed and implemented; a second audit in 2017 evaluated its impact.

Results: Standardised information was present on $77.5 \%$ of the magnetic resonance imaging (MRI) staging reports. Treatment strategies were similar in all three study centres. Of the patients whose treatment was interrupted, just 9.7\% received a compensation dose. There was an increase in MRI re-staging from 32.5 to $61.5 \%$, and a significant decrease in unreported circumferential resection margins following neoadjuvant therapy (ypCRM), from 34.5 to $5.6 \%(p<0.001)$.
\end{abstract}

Conclusions: The comparison between two clinical audits showed improvements in neoadjuvant radiotherapy in rectal cancer patients. Some indicators reveal areas in need of additional efforts, for example to reduce the overall treatment time.

Keywords: Rectal Cancer, Clinical audit, Radiotherapy process, Clinical practice variability, Improvement Quality process

\section{Introduction}

Colorectal cancer is the third most common cancer worldwide and the second in Catalonia; $45 \%$ of these tumours are in the rectum $[1,2]$. A locally advanced rectal tumour (LART) is defined by the presence of a neoplasm classified as T3/T4, with or without lymph node infiltration $(\mathrm{N} 0 / \mathrm{N}+)$, and no metastatic dissemination. Treatment is multidisciplinary and consists of surgery plus radiochemotherapy [3], with careful evaluation of

\footnotetext{
* Correspondence: gtorras@iconcologia.net

${ }^{1}$ Clinical Management Department, Institut Català d'Oncologia, Barcelona, Spain

Full list of author information is available at the end of the article
}

magnetic resonance imaging (MRI) parameters before and after preoperative treatment. The total mesorectal excision is a quality indicator, and the main factor for local control, but in LART the neoadjuvant treatment achieves greater control than surgery alone. Since the results of the Swedish Rectal Cancer Trial were published in 1997 [4], neoadjuvant, multimodal treatment has taken root as the standard treatment strategy. Substantial reductions in local recurrence have been achieved thanks to radiotherapy (RT), both short-course radiotherapy (SCRT) and long-course radiotherapy (LCRT), which is combined with chemotherapy. In historical series, local recurrence was 30 to $45 \%$, compared with

(C) The Author(s). 2020 Open Access This article is distributed under the terms of the Creative Commons Attribution 4.0 International License (http://creativecommons.org/licenses/by/4.0/), which permits unrestricted use, distribution, and 
less than $10 \%$ today [5]. In 2019, multimodality treatment for LART also implies personalised strategies to decrease the surgical burden for patients responding after pre-operative treatment, including 'wait and watch' or organ-preservation approaches.

Thus, successful treatment of locally advanced rectal cancer requires a multidisciplinary approach $[6,7]$. The complexity of care processes - and the resulting potential for variability in clinical decision-making - make monitoring highly desirable. Clinical audits, for their part, are a widely used instrument to identify variability in clinical practice and areas in need of improvement. Several countries have incorporated audits as a strategy to evaluate health policies $[8,9]$. The impact of the results obtained depends to a large extent on the analysis of the outcomes, the quality of the data collected, and the credibility of the audit team. There are few studies that report the effects of corrective measures established based on post-intervention audits [10].

The aim of this study was to assess the effects of the quality improvement action plan on the quality of clinical care processes in patients with rectal cancer, with an emphasis on the diagnostic and neoadjuvant treatment phases.

\section{Methods}

This study took place in the Institut Català d'Oncologia (ICO), a cancer care organisation with three specialised centres offering radiation oncology treatments. ICO collaborates with the corresponding university hospitals that belong to the Institut Calatà de la Salut (ICS);it works in a network with another 17 more local hospitals, and it is the reference centre in oncology for nearly 3 million people ( $45 \%$ of the adult population in Catalonia). The care model is characterised by a multidisciplinary focus on specific pathologies, in compliance with corporate clinical practice guidelines.

\section{Study design}

This multicentre comparative study was performed over two periods (2013 and 2017). For each study year, we selected a representative sample of patients diagnosed with rectal cancer and treated with neoadjuvant RT.

The study began with the creation of a working group made up of radiation oncologists and medical physicists from all three centres, and led by the clinical reference centre in rectal cancer. Key aspects of the diagnostic, therapeutic and follow-up phases were identified based on the literature $[11,12]$, as were the study indicators, including the appropriateness of the diagnostic tests performed, quality of the diagnostic reports, multidisciplinary approach, appropriateness and adherence to the prescribed treatment, imaging controls during the RT sessions, post-surgical circumferential resection margin
(ypCRM), and adverse effects. A purpose-designed form was used to collect the required data during the first audit, and for the second audit it was adapted to include the new indicators derived from the action plan.

The first clinical audit for radiation oncology (ACOR1) identified areas in need of improvement. The analysis of these published results [13] informed the development of an action plan, which was approved in April 2016 and implemented thereafter. A new audit (ACOR2) was then performed to assess the extent to which the improvement targets were achieved.

The audits consisted of a review of a random sample of 40 clinical histories from each centre. The target population was patients diagnosed with rectal cancer (International Classification of Diseases; ICD-9:154.1) who received neoadjuvant RT with a curative intent (starting in 2013 in the first cohort and between June and December of 2017 in the second).

For ACOR-2, the cognizant clinical practice guidelines specified the criteria for concomitant chemotherapy and two alternative strategies for RT: LCRT ( 45 Gy to 50.4 Gy in fractions of $1.8 \mathrm{~Gy}$ ) and SCRT (25 Gy in fractions of $5 \mathrm{~Gy}$ ), administered on 5 consecutive days. The SCRT strategy was preferentially indicated in patients over the age of 70, in tumours of the middle third of the rectum, and in disease without involvement of the mesorectal fascia or metastasis [14].

Following a pilot test, a team of external auditors undertook the first audit from June to September 2015, and the second from June to September 2018. Data were collected from the electronic clinical records in the participating centres (SAP i.s.h. med Cerner \& SAP) and the specific information system for radiotherapy (Aria oncology information System BY Varian Medical Systems Inc.).

\section{Action plan}

For the diagnostic phase, the quality improvement action plan (Table 1) covered measures to increase the available clinical information needed to develop a treatment strategy, based on personal communication and multidisciplinary committees. The audit highlighted the need to standardise the MRI report, based on the Mercury Study Group criteria [15]. Another aspect considered was tumour board review to approve the treatment plan, in patients diagnosed both in our centres and in the regional hospitals.

Measures to improve the treatment phase included standardising the use of intensity-modulated radiotherapy (IMRT) in the three centres, based on the following indications: tumours in the lower third of the rectum; patients with hysterectomy; rT4 rectal tumours; and infiltration to the prostate, bladder or uterus in patients in whom the planning target volume included the 
Table 1 Description of action plan (approved 18 April 2016)

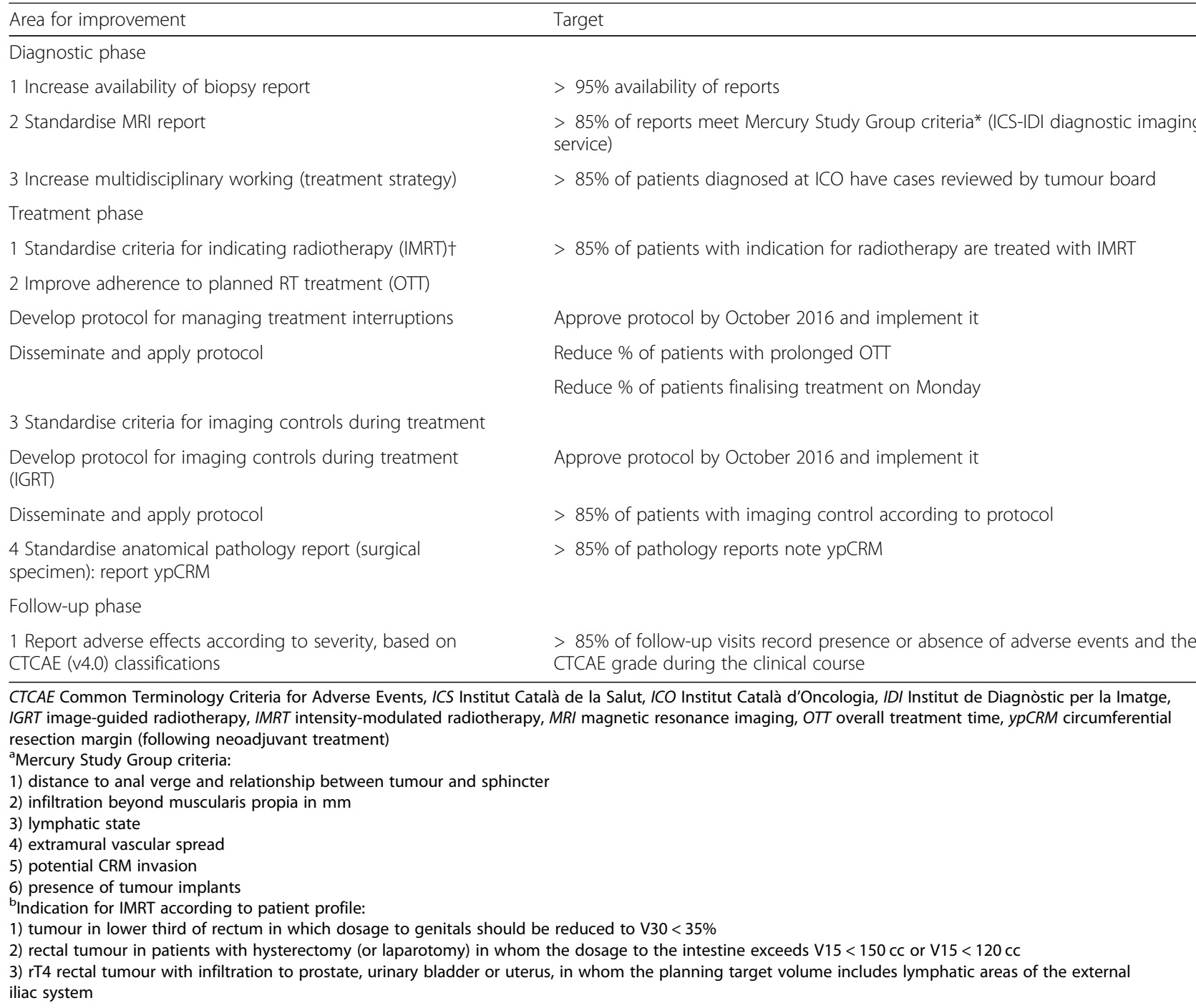

lymphatic areas of the external iliac system lymph nodes [16]. Other measures were the development and implementation of a management protocol for RT session interruptions to reduce prolonged overall treatment time (OTT) and a protocol for image-guided radiotherapy (IGRT) during the treatment (to be undertaken at least once a week); an increase in the percentage of patients re-evaluated with MRI prior to surgery; and the state of the ypCRM on the pathology report describing the surgical specimens.

Finally, for the follow-up, the action plan aimed to improve reporting of adverse effects over the clinical course, according to the Common Terminology Criteria for Adverse Events (CTCAE) v4.0 [17].

We used R software available as Free Software (version 3.0.2) for the statistical analysis. The sample size calculation was based on a level of confidence of $0.95(\alpha=$ 0.05 ), assuming a finite population of about 100 cases per centre and a $20 \%$ rate of attrition. Pearson's chisquared test was used to compare results between groups.

\section{Results}

Table 2 presents the characteristics of the patients included in the ACOR-1 and ACOR-2 cohorts. There were no significant differences in the demographic or clinical profiles, except in histological grade and distance from tumour to mesorectal fascia. Table 3 shows the outcomes for the diagnostic phase after implementing the action plan. Availability of the biopsy report reached $100 \%$ in patients in the second period, as did MRI staging in two of the three centres. The MRI reports met the established criteria in $77.5 \%$ of the cases, representing a significant rise with respect to the first period. In centre A, there were 10 cases where the report noted the distance from the 
Table 2 Patient's clinical characteristics

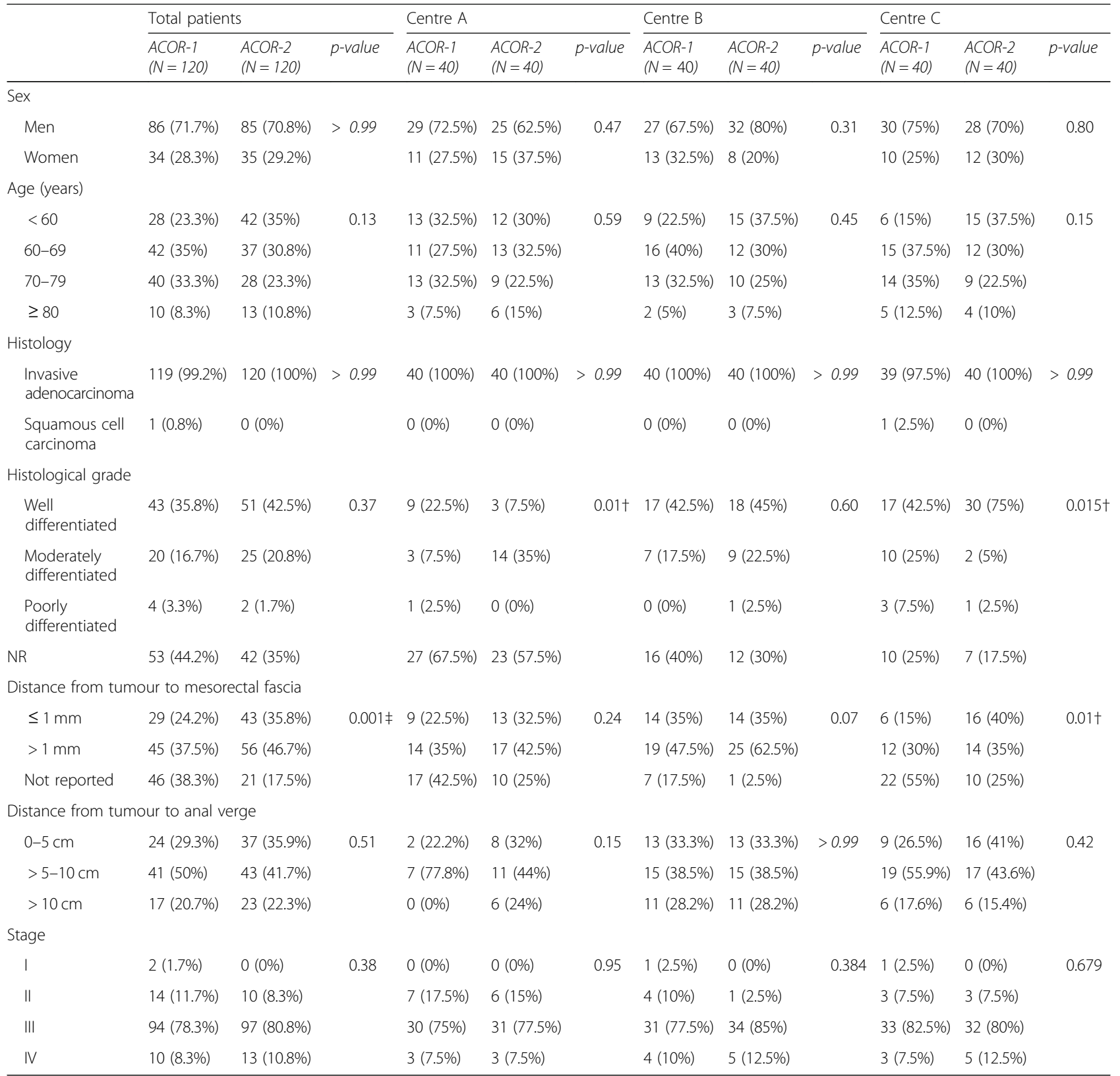

ACOR clinical audit for radiation oncology, ICS Institut Català de la Salut, ICO Institut Català d'Oncologia, NR not reported ${ }^{*} p$-value 0.05 to $0.01 ;+p$-value $\leq 0.01 ; \neq p$-value $\leq 0.001$

tumour to the anorectal junction rather than the anal verge (these measures are considered equivalent with respect to the fulfilment of the standard).

The outcomes for the treatment phase are detailed in Table 4. The pattern of neoadjuvant treatment is similar between centres and in both cohorts. In ACOR-2, 62.7\% of the patients received a total of 50.4 Gy (LCRT), a significant difference with respect to ACOR-1 that mainly reflects an improvement in centre $\mathrm{A}$.

A protocol for managing treatment interruptions was approved in June 2017. Compliance was 9.7\%; we did not observe a significant decrease in patients with prolonged OTT over seven days (in the case of LCRT) or in the percentage of patients who received their last dose on a Monday (radiotherapy units are not active on weekends). A protocol for image-guided radiotherapy was approved in October 2016; the compliance rate was $95.8 \%$.

There was a significant increase in the performance of the MRI restaging procedure in all centres, from 32.5 to $61.3 \%$ (Table 5). The proportion of patients in whom surgery was indicated dropped slightly, from 
Table 3 Action plan results: outcomes in diagnostic phase

\begin{tabular}{|c|c|c|c|c|c|c|c|c|c|c|c|c|}
\hline \multicolumn{4}{|c|}{ Total patients } & \multicolumn{3}{|l|}{ Centre A } & \multicolumn{3}{|l|}{ Centre B } & \multicolumn{3}{|l|}{ Centre C } \\
\hline & $\begin{array}{l}\text { ACOR-1 } \\
(N=120)\end{array}$ & $\begin{array}{l}\text { ACOR-2 } \\
(N=120)\end{array}$ & $p$-value & $\begin{array}{l}\text { ACOR-1 } \\
(N=40)\end{array}$ & $\begin{array}{l}A C O R-2 \\
(N=40)\end{array}$ & p-value & $\begin{array}{l}A C O R-1 \\
(N=40)\end{array}$ & $\begin{array}{l}\text { ACOR-2 } \\
(N=40)\end{array}$ & p-value & $\begin{array}{l}\text { ACOR-1 } \\
(N=40)\end{array}$ & $\begin{array}{l}\text { ACOR-2 } \\
(N=40)\end{array}$ & $\overline{p \text {-value }}$ \\
\hline \multicolumn{13}{|c|}{ Was the patient diagnosed outside of ICO-ICS? } \\
\hline Yes & $71(59.2 \%)$ & $100(83.3 \%)$ & $<0.001 \neq$ & $10(25 \%)$ & $29(72.5 \%)$ & $<0.001 \neq$ & $30(75 \%)$ & $37(92.5 \%)$ & $0.069^{*}$ & $31(77.5 \%)$ & $34(85 \%)$ & 0.57 \\
\hline No & $49(40.8 \%)$ & $20(16.7 \%)$ & & $30(75 \%)$ & $11(27.5 \%)$ & & $10(25 \%)$ & $3(7.5 \%)$ & & $9(22.5 \%)$ & $6(15 \%)$ & \\
\hline \multicolumn{13}{|c|}{ In patients diagnosed in ICO-ICS, did a tumour board review the case? } \\
\hline Yes & 39 (88.6\%) & $20(100 \%)$ & 0.29 & $25(86.2 \%)$ & $11(100 \%)$ & 0.48 & $10(100 \%)$ & $3(100 \%)$ & 0.052 & $4(80 \%)$ & $6(100 \%)$ & 0.92 \\
\hline No & $5(11.4 \%)$ & $0(0 \%)$ & & $4(13.8 \%)$ & $0(0 \%)$ & & $0(0 \%)$ & $0(0 \%)$ & & $1(20 \%)$ & $0(0 \%)$ & \\
\hline \multicolumn{13}{|c|}{ In patients diagnosed outside of ICO-ICS, did a tumour board review the case? } \\
\hline Yes & $34(47.9 \%)$ & $68(68 \%)$ & $0.019^{*}$ & $3(30 \%)$ & $17(58.6 \%)$ & 0.23 & $18(60 \%)$ & 35 (94.6\%) & 0.002 & $13(41.9 \%)$ & $16(47.1 \%)$ & 0.55 \\
\hline No & $36(50.7 \%)$ & $32(32 \%)$ & & $7(70 \%)$ & $12(41.4 \%)$ & & $12(40 \%)$ & $2(5.4 \%)$ & & $17(54.8 \%)$ & $18(52.9 \%)$ & \\
\hline NR & $1(1.4 \%)$ & $0(0 \%)$ & & $0(0 \%)$ & $0(0 \%)$ & & $0(0 \%)$ & $0(0 \%)$ & & $1(3.2 \%)$ & $0(0 \%)$ & \\
\hline \multicolumn{13}{|c|}{ Was the biopsy report in the medical history? } \\
\hline Yes & $110(91.7 \%)$ & $120(100 \%)$ & $0.004 \dagger$ & $37(92.5 \%)$ & $40(100 \%)$ & 0.24 & 37 (92.5\%) & $40(100 \%)$ & 0.24 & $36(90 \%)$ & $40(100 \%)$ & 0.12 \\
\hline No & $10(8.3 \%)$ & $0(0 \%)$ & & $3(7.5 \%)$ & $0(0 \%)$ & & $3(7.5 \%)$ & $0(0 \%)$ & & $4(10 \%)$ & $0(0 \%)$ & \\
\hline \multicolumn{13}{|c|}{ Was the pelvic MRI recorded in the diagnostic report? } \\
\hline Yes & $114(95 \%)$ & $119(99.2 \%)$ & 0.13 & $38(95 \%)$ & $40(100 \%)$ & 0.47 & $40(100 \%)$ & $40(100 \%)$ & $>0.99$ & $36(90 \%)$ & $39(97.5 \%)$ & 0.36 \\
\hline No & $6(5 \%)$ & $1(0.8 \%)$ & & $2(5 \%)$ & $0(0 \%)$ & & $0(0 \%)$ & $0(0 \%)$ & & $4(10 \%)$ & $1(2.5 \%)$ & \\
\hline \multicolumn{13}{|c|}{ Did the MRI report include information on the distance to the anal verge or to the anorectal union**? } \\
\hline Yes & $82(68.3 \%)$ & $116(96.7 \%)$ & $<0.001^{\ddagger}$ & $9(22.5 \%)$ & $38(95 \%)$ & $0.001 \neq$ & $39(97.5 \%)$ & $39(97.5 \%)$ & $>0.99$ & $34(85 \%)$ & $39(97.5 \%)$ & 0.12 \\
\hline No & $38(31.7 \%)$ & $4(3.3 \%)$ & & $31(77.5 \%)$ & $2(5 \%)$ & & $1(2.5 \%)$ & $1(2.5 \%)$ & & $6(15 \%)$ & $1(2.5 \%)$ & \\
\hline \multicolumn{13}{|c|}{ Did the MRI report include information on the distance to the mesorectal fascia? } \\
\hline Yes & $74(61.7 \%)$ & $99(82.5 \%)$ & $<0.001^{\ddagger}$ & $23(57.5 \%)$ & $30(75 \%)$ & 0.16 & $33(82.5 \%)$ & $39(97.5 \%)$ & 0.062 & $18(45 \%)$ & $30(75 \%)$ & $0.012^{*}$ \\
\hline No & $46(38.3 \%)$ & $21(17.5 \%)$ & & $17(42.5 \%)$ & $10(25 \%)$ & & $7(17.5 \%)$ & $1(2.5 \%)$ & & $22(55 \%)$ & $10(25 \%)$ & \\
\hline \multicolumn{13}{|c|}{ Did the MRI report include enough information to assign a 'T' value? } \\
\hline Yes & $112(93.3 \%)$ & $117(98.3 \%)$ & 0.11 & $37(92.5 \%)$ & $39(97.5 \%)$ & 0.61 & $39(97.5 \%)$ & $40(100 \%)$ & $>0.99$ & $36(90 \%)$ & $38(97.4 \%)$ & 0.37 \\
\hline No & $8(6.7 \%)$ & $2(1.7 \%)$ & & $3(7.5 \%)$ & $1(2.5 \%)$ & & $1(2.5 \%)$ & $0(0 \%)$ & & $4(10 \%)$ & $1(2.6 \%)$ & \\
\hline \multicolumn{13}{|c|}{ Did the MRI report include enough information to assign an ' $N$ ' value? } \\
\hline Yes & $120(100 \%)$ & $118(99.2 \%)$ & $>0.99$ & $40(100 \%)$ & $39(97.5 \%)$ & $>0.99$ & $40(100 \%)$ & $40(100 \%)$ & $>0.99$ & $40(100 \%)$ & $39(100 \%)$ & 0.91 \\
\hline No & $0(0 \%)$ & $1(0.8 \%)$ & & $0(0 \%)$ & $1(2.5 \%)$ & & $0(0 \%)$ & $0(0 \%)$ & & $0(0 \%)$ & $0(0 \%)$ & \\
\hline \multicolumn{13}{|c|}{ Did the MRI report meet Mercury Study Group criteria? } \\
\hline Yes & $54(45 \%)$ & $93(77.5 \%)$ & $<0.001 \neq$ & $5(12.5 \%)$ & $27(67.5 \%)$ & $<0.001 \neq$ & $33(82.5 \%)$ & $38(95 \%)$ & 0.16 & $16(40 \%)$ & $28(70 \%)$ & $0.013^{*}$ \\
\hline No & $66(55 \%)$ & $27(22.5 \%)$ & & $35(87.5 \%)$ & $13(32.5 \%)$ & & $7(17.5 \%)$ & $2(5 \%)$ & & $24(60 \%)$ & $12(30 \%)$ & \\
\hline
\end{tabular}

ACOR clinical audit for radiation oncology, ICS Institut Català de la Salut, ICO Institut Català d'Oncologia, MRI magnetic resonance imaging, NR not reported ${ }^{*} p$-value 0.05 to $0.01 ; t p$-value $\leq 0.01 ; \neq p$-value $\leq 0.001$

${ }^{* *}$ Centre A: 13 cases had the distance to the anorectal union (not to the anal verge)

96.7 to $90 \%$, reflecting an increase in patients following a conservative treatment strategy. There was also a significant improvement in the availability of the ypCRM on the anatomical pathology report (ACOR-2: 94.4\%).

For the follow-up phase, adverse effects of treatment were reported on $99.8 \%$ of the visits, and these were graded for severity according to the CTCAE in $36.2 \%$ of the cases.

\section{Discussion}

Clinical audits are a well-known tool for identifying areas to improve in the care process. This study reports the results of an action plan for improving quality in neoadjuvant treatment for locally advanced rectal cancer in three centres, based on the findings of an initial quality audit. The ACOR-1 audit revealed variability in clinical practice between centres and showed suboptimal outcomes for several standard quality indicators. ACOR- 
Table 4 Action plan results: outcomes in treatment phase I (neoadjuvant treatment)

\begin{tabular}{|c|c|c|c|c|c|c|c|c|c|c|c|c|}
\hline & \multicolumn{3}{|c|}{ Total patients } & \multicolumn{3}{|l|}{ Centre A } & \multicolumn{3}{|l|}{ Centre B } & \multicolumn{3}{|l|}{ Centre C } \\
\hline & $\begin{array}{l}\text { ACOR-1 } \\
(N=120)\end{array}$ & $\begin{array}{l}\text { ACOR-2 } \\
(N=120)\end{array}$ & $p$-value & $\begin{array}{l}\text { ACOR-1 } \\
(\mathrm{N}=40)\end{array}$ & $\begin{array}{l}\text { ACOR-2 } \\
(\mathrm{N}=40)\end{array}$ & $p$-value & $\begin{array}{l}\text { ACOR-1 } \\
(N=120)\end{array}$ & $\begin{array}{l}\text { ACOR-2 } \\
(N=120)\end{array}$ & $\begin{array}{l}p- \\
\text { value }\end{array}$ & $\begin{array}{l}\text { ACOR-1 } \\
(N=40)\end{array}$ & $\begin{array}{l}\text { ACOR-2 } \\
(N=40)\end{array}$ & $\begin{array}{l}p- \\
\text { value }\end{array}$ \\
\hline \multicolumn{13}{|c|}{ Planned treatment } \\
\hline $\begin{array}{l}\text { RT }+ \\
\text { chemo }\end{array}$ & $115(95.8 \%)$ & 107 (89.2\%) & 0.086 & $37(92.5 \%)$ & $34(85 \%)$ & 0.48 & $39(97.5 \%)$ & $39(97.5 \%)$ & $>0.99$ & 39 (97.5\%) & $34(85 \%)$ & 0.11 \\
\hline RT & $5(4.2 \%)$ & $13(10.8 \%)$ & & $3(7.5 \%)$ & $6(15 \%)$ & & $1(2.5 \%)$ & $1(2.5 \%)$ & & $1(2.5 \%)$ & $6(15 \%)$ & \\
\hline \multicolumn{13}{|c|}{ Type of radiotherapy } \\
\hline LCRT & $108(90 \%)$ & $102(85 \%)$ & 0.33 & $36(90 \%)$ & $32(80 \%)$ & 0.35 & $40(100 \%)$ & $38(95 \%)$ & 0.47 & $32(80 \%)$ & $32(80 \%)$ & $>0.99$ \\
\hline SCRT & $12(10 \%)$ & $18(15 \%)$ & & $4(10 \%)$ & $8(20 \%)$ & & $0(0 \%)$ & $2(5 \%)$ & & $8(20 \%)$ & $8(20 \%)$ & \\
\hline \multicolumn{13}{|c|}{ Type of indicated technology } \\
\hline $3 \mathrm{D}$ & $117(97.5 \%)$ & 95 (79.2\%) & $<0.001 \neq$ & $40(100 \%)$ & $23(57.5 \%)$ & $<0.001 \neq$ & $40(100 \%)$ & $38(95 \%)$ & 0.47 & 37 (92.5\%) & $34(85 \%)$ & 0.48 \\
\hline VMAT & $3(2.5 \%)$ & $25(20.8 \%)$ & & $0(0 \%)$ & $17(42.5 \%)$ & & $0(0 \%)$ & $2(5 \%)$ & & $3(7.5 \%)$ & $6(15 \%)$ & \\
\hline \multicolumn{13}{|c|}{ Total dosage administered } \\
\hline $25 \mathrm{~Gy}$ & $12(10.3 \%)$ & $18(15.3 \%)$ & $0.034^{*}$ & $4(10.3 \%)$ & $8(21.1 \%)$ & $<0.001 \neq$ & $0(0 \%)$ & $2(5 \%)$ & 0.36 & $8(21.1 \%)$ & $8(20 \%)$ & 0.57 \\
\hline $45 \mathrm{~Gy}$ & $34(29.1 \%)$ & 15 (12.7\%) & & $34(87.2 \%)$ & $14(36.8 \%)$ & & $0(0 \%)$ & $0(0 \%)$ & & $0(0 \%)$ & $1(2.5 \%)$ & \\
\hline 50 Gy & $3(2.6 \%)$ & $6(5.1 \%)$ & & $0(0 \%)$ & $0(0 \%)$ & & $0(0 \%)$ & $0(0 \%)$ & & $3(7.9 \%)$ & $6(15 \%)$ & \\
\hline $50.4 \mathrm{~Gy}$ & $63(53.8 \%)$ & $74(62.7 \%)$ & & $1(2.6 \%)$ & $16(42.1 \%)$ & & $35(87.5 \%)$ & $33(82.5 \%)$ & & $27(71.1 \%)$ & $25(62.5 \%)$ & \\
\hline $54 \mathrm{~Gy}$ & $5(4.3 \%)$ & $5(4.2 \%)$ & & $0(0 \%)$ & $0(0 \%)$ & & $5(12.5 \%)$ & $5(12.5 \%)$ & & $0(0 \%)$ & $0(0 \%)$ & \\
\hline \multicolumn{13}{|c|}{ Prolongation of treatment time (if SCRT) } \\
\hline$<3$ days & $12(100 \%)$ & $17(94.4 \%)$ & $>0.99$ & $4(100 \%)$ & $8(100 \%)$ & 0.25 & 0 (NaN\%) & $2(100 \%)$ & 0.16 & $8(100 \%)$ & $7(87.5 \%)$ & $>0.99$ \\
\hline $3-5$ days & $0(0 \%)$ & $1(5.6 \%)$ & & $0(0 \%)$ & $0(0 \%)$ & & $\mathrm{O}(\mathrm{NaN} \%)$ & $0(0 \%)$ & & $0(0 \%)$ & $1(12.5 \%)$ & \\
\hline \multicolumn{13}{|c|}{ Prolongation of treatment time (if LCRT) } \\
\hline$<3$ days & $62(57.4 \%)$ & $58(56.9 \%)$ & 0.96 & $19(52.8 \%)$ & $20(62.5 \%)$ & 0.22 & $31(77.5 \%)$ & $27(71.1 \%)$ & 0.53 & $12(37.5 \%)$ & $11(34.4 \%)$ & 0.90 \\
\hline $3-5$ days & $32(29.6 \%)$ & 31 (30.4\%) & & $11(30.6 \%)$ & $10(31.2 \%)$ & & $9(22.5 \%)$ & $9(23.7 \%)$ & & $12(37.5 \%)$ & $12(37.5 \%)$ & \\
\hline $6-7$ days & $4(3.7 \%)$ & $5(4.9 \%)$ & & $0(0 \%)$ & $1(3.1 \%)$ & & $0(0 \%)$ & $1(2.6 \%)$ & & $4(12.5 \%)$ & $3(9.4 \%)$ & \\
\hline$>7$ days & $10(9.3 \%)$ & $8(7.8 \%)$ & & $6(16.7 \%)$ & $1(3.1 \%)$ & & $0(0 \%)$ & $1(2.6 \%)$ & & $4(12.5 \%)$ & $6(18.8 \%)$ & \\
\hline \multicolumn{13}{|c|}{ Week day when treatment was finalised } \\
\hline Monday & $17(14.2 \%)$ & $7(5.8 \%)$ & 0.24 & $6(15 \%)$ & $1(2.5 \%)$ & 0.16 & $6(15 \%)$ & $3(7.5 \%)$ & 0.68 & $5(12.5 \%)$ & $3(7.5 \%)$ & 0.28 \\
\hline Tuesday & $24(20 \%)$ & $22(18.3 \%)$ & & $11(27.5 \%)$ & $11(27.5 \%)$ & & $5(12.5 \%)$ & $6(15 \%)$ & & $8(20 \%)$ & $5(12.5 \%)$ & \\
\hline Wednesday & $13(10.8 \%)$ & $12(10 \%)$ & & $5(12.5 \%)$ & $5(12.5 \%)$ & & $2(5 \%)$ & $1(2.5 \%)$ & & $6(15 \%)$ & $6(15 \%)$ & \\
\hline Thursday & $24(20 \%)$ & $27(22.5 \%)$ & & $8(20 \%)$ & $5(12.5 \%)$ & & $11(27.5 \%)$ & $9(22.5 \%)$ & & $5(12.5 \%)$ & $13(32.5 \%)$ & \\
\hline Friday & $42(35 \%)$ & $52(43.3 \%)$ & & $10(25 \%)$ & $18(45 \%)$ & & $16(40 \%)$ & 21 (52.5\%) & & $16(40 \%)$ & $13(32.5 \%)$ & \\
\hline
\end{tabular}

ACOR clinical audit for radiation oncology, LCRT long-course radiotherapy, SCRT short-course radiotherapy, $R T$ radiotherapy, VMAT volumetric modulated arc therapy ${ }^{*} p$-value 0.05 to $0.01 ; \uparrow p$-value $\leq 0.01 ; \neq p$-value $\leq 0.001$

2 showed the evolution of these indicators following the implementation of quality improvement measures.

In the diagnostic phase, the MRI is the best tool to identify the patients with locally advanced tumours who would most benefit from neoadjuvant treatment [14]. All the cases audited in ACOR-2 included this test, and there was a significant improvement in the quality of the report. Specifically, there was an increase in adherence to the Mercury Group's recommendations on reporting rectal cancer MRIs [15], including the distance from the tumour to the mesorectal fascia (CRM), reported in 61.7\% of the cases in ACOR-1 and 82.5\% in ACOR-2, and the distance to the anal verge or the anorectal union, reported in 68.3 and $96.7 \%$ of the cases, respectively $(p<0.001)$. Overall, the centres did not achieve the optimal level of reporting (85\%), but $77.5 \%$ of the reports in ACOR-2 did meet the established criteria, compared to just $45 \%$ in ACOR-1 - a significant change. We consider that having a proforma, which would include the state of the resection margin (CRM) and the distance from the tumour to the anal verge, would be a decisive factor in achieving optimal reporting, as described by Taylor and colleagues $[18,19]$.

The number of patients coming to the ICO-ICS unit with a diagnosis in hand increased significantly, probably due to the centralisation policies implemented for this 
Table 5 Action plan results: outcomes in treatment phase II (restaging and surgical treatment)

\begin{tabular}{|c|c|c|c|c|c|c|c|c|c|c|c|c|}
\hline & \multicolumn{3}{|c|}{ Total patients } & \multicolumn{3}{|l|}{ Centre A } & \multicolumn{3}{|l|}{ Centre B } & \multicolumn{3}{|l|}{ Centre C } \\
\hline & $\begin{array}{l}\text { ACOR-1 } \\
(N=120)\end{array}$ & $\begin{array}{l}\text { ACOR-2 } \\
(N=120)\end{array}$ & $p$-value & $\begin{array}{l}A C O R-1 \\
(N=40)\end{array}$ & $\begin{array}{l}\text { ACOR-2 } \\
(N=40)\end{array}$ & $p$-value & $\begin{array}{l}\text { ACOR-1 } \\
(N=120)\end{array}$ & $\begin{array}{l}\text { ACOR-2 } \\
(N=120)\end{array}$ & $\overline{p \text {-value }}$ & $\begin{array}{l}\text { ACOR-1 } \\
(N=40)\end{array}$ & $\begin{array}{l}\text { ACOR-2 } \\
(N=40)\end{array}$ & $\overline{p \text {-value }}$ \\
\hline \multicolumn{13}{|c|}{ Was the restaging report available? } \\
\hline Yes & $39(32.5 \%)$ & $73(61.3 \%)$ & $<0.001 \neq$ & $0(0 \%)$ & $16(40 \%)$ & $<0.001 \neq$ & $31(77.5 \%)$ & 37 (94.9\%) & 0.057 & $8(20 \%)$ & $20(50 \%)$ & $0.01+$ \\
\hline No & $81(67.5 \%)$ & $46(38.7 \%)$ & & $40(100 \%)$ & $24(60 \%)$ & & $9(22.5 \%)$ & $2(5.1 \%)$ & & $32(80 \%)$ & $20(50 \%)$ & \\
\hline \multicolumn{13}{|c|}{ Did the patient undergo surgery? } \\
\hline Yes & $116(96.7 \%)$ & $108(90 \%)$ & $0.035^{*}$ & $40(100 \%)$ & $33(82.5 \%)$ & $0.018^{*}$ & $37(92.5 \%)$ & 37 (92.5\%) & $>0.99$ & $39(97.5 \%)$ & $38(95 \%)$ & 0.22 \\
\hline No & $3(2.5 \%)$ & $12(10 \%)$ & & $0(0 \%)$ & $7(17.5 \%)$ & & $3(7.5 \%)$ & $3(7.5 \%)$ & & $0(0 \%)$ & $2(5 \%)$ & \\
\hline NR & $1(0.8 \%)$ & $0(0 \%)$ & & $0(0 \%)$ & $0(0 \%)$ & & $0(0 \%)$ & $0(0 \%)$ & & 1 (2.5\%) & $0(0 \%)$ & \\
\hline \multicolumn{13}{|c|}{ Staging (in patients undergoing surgical treatment) } \\
\hline 0 & 19 (16.4\%) & 20 (18.5\%) & 0.54 & 7 (17.5\%) & 5 (15.2\%) & 0.52 & 7 (18.9\%) & $9(24.3 \%)$ & 0.93 & $5(12.8 \%)$ & $6(15.8 \%)$ & 0.53 \\
\hline । & $4(3.4 \%)$ & $3(2.8 \%)$ & & $2(5 \%)$ & $1(3 \%)$ & & $1(2.7 \%)$ & $1(2.7 \%)$ & & $1(2.6 \%)$ & $1(2.6 \%)$ & \\
\hline$\|$ & 62 (53.4\%) & 47 (43.5\%) & & $20(50 \%)$ & 12 (36.4\%) & & $18(48.6 \%)$ & 19 (51.4\%) & & 24 (61.5\%) & $16(42.1 \%)$ & \\
\hline III & $25(21.6 \%)$ & 28 (25.9\%) & & $10(25 \%)$ & 12 (36.4\%) & & $9(24.3 \%)$ & $6(16.2 \%)$ & & $6(15.4 \%)$ & $10(26.3 \%)$ & \\
\hline IV & $6(5.2 \%)$ & $10(9.3 \%)$ & & $1(2.5 \%)$ & $3(9.1 \%)$ & & $2(5.4 \%)$ & $2(5.4 \%)$ & & $3(7.7 \%)$ & 5 (13.2\%) & \\
\hline \multicolumn{13}{|c|}{ ypCRM (in patients undergoing surgical treatment) } \\
\hline Positive & $5(4.3 \%)$ & $16(14.8 \%)$ & $<0.001 \neq$ & $1(2.5 \%)$ & $4(12.1 \%)$ & $<0.001 \neq$ & $3(8.1 \%)$ & $3(8.1 \%)$ & $0.037^{*}$ & $1(2.6 \%)$ & $9(23.7 \%)$ & $0.006+$ \\
\hline Negative & $71(61.2 \%)$ & $86(79.6 \%)$ & & $15(37.5 \%)$ & $26(78.8 \%)$ & & $28(75.7 \%)$ & $34(91.9 \%)$ & & $28(71.8 \%)$ & $26(68.4 \%)$ & \\
\hline NR & 40 (34.5\%) & $6(5.6 \%)$ & & $24(60 \%)$ & 3 (9.1\%) & & $6(16.2 \%)$ & $0(0 \%)$ & & $10(25.6 \%)$ & $3(7.9 \%)$ & \\
\hline
\end{tabular}

ACOR clinical audit for radiation oncology, $N R$ not reported

${ }^{*} p$-value 0.05 to $0.01 ; \dagger p$-value $\leq 0.01 ; \neq p$-value $\leq 0.001$

pathology in the region [20]. All the cases diagnosed in the ICO unit were reviewed by a tumour board to approve the therapeutic strategy.

In ACOR-2, the treatment plan adopted most frequently was radiochemotherapy, with LCRT and a dose of $50.4 \mathrm{~Gy}$. Compared with the outcomes observed in ACOR-1, ACOR-2 showed greater standardisation between centres and better adherence to the approved guidelines, although some variability persists, which is consistent with other studies [21]. For example, two of the centres used SCRT in $20 \%$ of the patients, while the other did so in just $5 \%$. This difference is probably due to the divergent approaches in patients of advanced age. One centre adheres to the strategy for avoiding surgery in frail patients, so its adoption of LCRT is more assiduous. In contrast, the other two centres favour the avoidance of chemotherapy [22, 23].

We also noted an increase in the use of volumetric modulated arc therapy (VMAT), from 2.5 to $20.8 \%$ $(p<0.001)$, and its dissemination in all three centres. Although the radiated intestinal volume may be susceptible to late toxicity, VMAT reduces acute toxicity and consequently, treatment interruptions [16]. Thus, consensus-based indications were developed for patients with tumours that infiltrated into adjacent organs, and in general when treatment of the external iliac lymph nodes and/or the groin nodes is unavoidable.
One of the action plan's target areas was OTT. Even though special efforts were made to improve this indicator, both audits showed that the overall RT treatment time was still more than seven days longer than planned for LCRT, usually because of weekday public holidays and technical inspections of the accelerators. There were no significant changes between study periods. We also observed a non-significant improvement in the percentage of patients whose last RT session was on a Monday. To improve this aspect, a protocol for managing treatment interruptions was designed and finally approved in June 2017. The delay in approval is probably the reason that we identified only seven patients in whom it was appropriately applied (four of them in centre B). Indeed, the included patients initiated treatment only from June to December 2017. Thus, the centres still have work to do in improving adherence to the treatment. As with any measure that affects the daily work routine and entails organisational changes, achieving success in this endeavour will be challenging [24].

Another target area was the standardisation of IGRT during treatment. This measure, too, was subject to a new protocol (approved in October 2016), and adherence was $95.8 \%$.

Once the neoadjuvant treatment is finalised, restaging (primarily based on MRI) is necessary to define the surgical technique. We observed a significant rise in the 
adoption of this approach in all three centres, with $32.5 \%$ of all cases undergoing MRI restaging in ACOR-1 and $61.3 \%$ in ACOR-2 $(p<0.001)$. Moreover, there is already a consolidated scientific basis permitting selected patients to follow a conservative strategy and avoid surgery, that is, 'watch and wait' $[25,26]$. In our case, in ACOR-2, surgery was indicated in $90 \%$ of the patients, compared to $96.7 \%$ in ACOR-1 $(p=0.035)$. In $3 / 12$ patients $(25 \%)$ in whom surgery was not indicated, the reason was having $\mathrm{CT}_{0} \mathrm{~N}_{0}$ after completing chemoradiotherapy.

Without a doubt, the most important quality indicator in neoadjuvant treatment of rectal cancer for local control and survival is the state of the circumferential resection margin (ypCRM). This detail was not described in the anatomical pathology report for $34.5 \%$ of the surgical specimens in ACOR-1, compared to $5.6 \%$ in ACOR-2 $(p<0.001)$. Overall, $14.8 \%$ of the surgical specimens showed CRM involvement (ypCRM+), which is consistent with the literature $[27,28]$. Considering the RT strategy, $13 \%$ of the LCRT treatments had ypCRM+, compared to $25 \%$ of those with SCRT treatment. We considered the proportion of $10 \%$ to be optimal.

With regard to the low-quality reporting of adverse events over the clinical course (absence of grading and aspects related to sexual function or bowel control), the specific list of CTCAE ( $\mathrm{v}$ 4.0) criteria [15] helped to moderately improve reporting. Nevertheless, the results obtained in ACOR-2 highlight the need to double down on efforts to improve this aspect.

The ultimate aim of treatment is not only to cure disease, but also to restore the person's dignity and achieve their social reinsertion. To this end, it is essential to understand the impact of dysfunctions and symptoms derived from treatment on patients' quality of life [29]. Future studies are necessary to explore this aspect in the long term, applying specific validated instruments like the EORTC questionnaires for quality of life in cancer patients and for colorectal quality of life in patients with rectal cancer receiving neoadjuvant treatment.

This study has several limitations. Some are related to the design of the audit, which included only 40 clinical histories per centre, limiting the power of the assessment, especially for clinical outcomes. In addition, the period selected (especially for the ACOR-2) allowed little time for the consolidation of changes derived from the action plan. Moreover, the data collected were from clinical histories; any measure taken but not recorded on the patient's electronic medical record would, for the purposes of the clinical audit, be non-existent.

In conclusion, this study analyses the influence of feedback from clinical audit data in improving neoadjuvant treatment procedures in rectal cancer. Our results show progress as well as areas in which additional efforts should be made, including: decreasing the percentage of positive ypCRMs; limiting treatment interruptions in order to reduce overall treatment time; and better selection of therapeutic options in elderly patients, according to frailty and mobility. In short, the audit undertaken shows total or partial correction in most areas targeted for quality improvements following the first audit, a decrease in variability between centres, and an improvement in adherence to clinical practice guidelines for rectal cancer.

\section{Abbreviations \\ ACOR-1: first clinical audit for radiation oncology; ACOR-2: second clinical audit for radiation oncology; CTCAE: Common Terminology Criteria for Adverse Events; EORTC: European Organisation for Research and Treatment of Cancer; ICO: Institut Català d'Oncologia; ICS: Institut Català de la Salut; IGRT: image-guided radiotherapy; IMRT: intensity-modulated radiotherapy; LART: locally advanced rectal tumour; LCRT: long course radiotherapy; MRI: magnetic resonance imaging; OTT: overall treatment time; $\mathrm{RT}$ : radiotherapy; SCRT: short course radiotherapy; VMAT: volumetric modulated arc therapy; ypCRM: circumferential resection margin (following neoadjuvant treatment)}

\section{Acknowledgments}

We would like to thank all the professionals involved in the radiotherapy process in the Institut Català d'Oncologia (Catalonia), without whom this study would not have been possible.

\section{Authors' contributions}

Involved in the original idea for the research: GF, VA, BJM, TMG. Promotion of the project and resources offered: VA, BJM, GF. Member of the ACOR group (process plan, key áreas of results, indicators, analysis of the results, proposal for the improvement plan): TMG, CE, MMC, JD, EA, VS, CM, MJ, MM, GF. Coordinator of the ACOR group in charge of the clinical audit design: TMG. To undertake the collection of data: PM, Process of case selection of the sample: MMC, MJ, GME, PM. Preparation of the improvement plan and implementation of it at each center: TMG, CE, MMC, JD, EA, VS, CM, MJ, MM, GF. Statistical analysis and clinical audit methodology recommendations: LA. Responsibility for the first draft of the manuscript: TMG. Revision of the first draft of the manuscript by the authors: All authors. Read and approved the final manuscript: All authors. Final corrections of the draft manuscript: BJM.

\section{Funding}

All funding or project support is acknowledged in the manuscript.

Availability of data and materials

The datasets during and/or analysed during the current study available from the corresponding author on reasonable request.

\section{Ethics approval and consent to participate}

All procedures performed in studies involving human participants were in accordance with the ethical standards of the institutional and/or national research committee (Comité Ético de Investigación Clínica del Hospital de Bellvitge, Ref. PR015/15) and with the 1964 Helsinki declaration and its later amendments or comparable ethical standards.

Consent for publication

Not applicable.

\section{Competing interests}

The authors declare that they have no competing interests.

\section{Author details}

${ }^{1}$ Clinical Management Department, Institut Català d'Oncologia, Barcelona, Spain. ${ }^{2}$ Radiation Oncology Department, Institut Català d'Oncologia, Girona, Spain. ${ }^{3}$ Medical Physics and Radiation Protection Department, Institut Català d'Oncologia, Girona, Spain. ${ }^{4}$ Quality and Results Department, Institut Català d'Oncologia, Girona, Spain. ${ }^{5}$ Medical Physics and Radiation Protection 
Department, Institut Català d'Oncologia, Girona, Spain. ${ }^{6}$ Radiation Oncology Department, Institut Català d'Oncologia, Badalona, Spain. ${ }^{7}$ Medical Physics and Radiation Protection Department, Institut Català d'Oncologia, Girona, Spain. ${ }^{8}$ Radiation Oncology Department, Institut Català d'Oncologia, Hospitalet del Llobregat, Barcelona, Spain. ${ }^{9}$ Hospital Tumor Registry, Institut Català d'Oncologia, Girona, Spain. ${ }^{10}$ Cancer Prevention and Control Program, Institut Català d'Oncologia, Hospitalet del Llobregat, Barcelona, Spain.

${ }^{11}$ Radiation Oncology Department, Institut Català d'Oncologia, Barcelona, Spain. ${ }^{12}$ Department of Clinical Sciences, IDIBELL, University of Barcelona, Barcelona, Spain.

\section{Received: 18 October 2019 Accepted: 14 January 2020}

\section{Published online: 31 January 2020}

\section{References}

1. Vernet R, Borras JM, Aliste L, Antonio M, Guarga A, Manchon-Walsh P. Influence of age on variation in patterns of care in patients with rectal cancer in Catalonia (Spain). Clin Transl Oncol. 2018;20:1538.

2. Ferlay J, Steliarova-Foucher E, Lortet-Tieulent J, Rosso S, Coebergh JW, Comber H, Forman D, Bray F. Cancer incidence and mortality patterns in Europe: estimates for 40 countries in 2012. Eur J Cancer. 2013;49:1374-403.

3. Rich T, Gunderson LL, Lew R, Galdibini JJ, Cohen AM, Donaldson G. Patterns of recurrence of rectal cancer after potentially curative surgery. Cancer. 1983;52:1317-29.

4. Kapiteijn E, Marijnen CA, Nagtegaal ID, Putter H, Steup WH, Wiggers T. Et Al. preoperative radiotherapy combined with total mesorectal excision for resectable rectal cancer. N Engl J Med. 2001;345(9):638-46.

5. MacFarlane JK, Ryall RD, Heald RJ. Mesorectal excision for rectal cancer. Lancet. 1993;341:457-60.

6. Lee M, Gibbs P, Wong R. Multidisciplinary Management of Locally Advanced Rectal Cancer-an Evolving Landscape? Clin Colorectal Cancer. 2015;14(4):251-61.

7. van de Velde CJ, Boelens PG, Borras JM, Coebergh JW, Cervantes A, Blomqvist $L$, et al. EURECCA colorectal: multidisciplinary management: European consensus conference colon \& rectum. Eur J Cancer. 2014;50(1):1.e1-1.e34.

8. Taylor A, Neuburger J, Walker K, Cromwell D, Groene O. How is feedback from national clinical audits used? Views from English National Health Service trust audit leads. J Health Serv Res Policy. 2016;21(2):91-100.

9. Prades J, Manchon-Walsh P, Solà J, Espinàs JA, Guarga A, Borras JM. Improving clinical outcomes through centralization of rectal cancer surgery and clinical audit: a mixed-methods assessment. Eur J Pub Health. 2016; 26(4):538-42.

10. Zerillo JA, Schouwenburg MG, van Bommel ACM, Stowell C, Lippa J, et al. An international collaborative standardizing a comprehensive patientcentered outcomes measurement set for colorectal cancer. JAMA Oncol. 2017;3(5):686-94

11. Manchon-Walsh P, Borras JM, Espinas JA, Aliste L. On behalf of the Catalonian rectal Cancer group. Et al. variability in the quality of rectal cancer care in public hospitals in Catalonia (Spain): clinical audit as a basis for action. Eur J Surg Oncol. 2011;37(4):325-33.

12. Fundowicz M, Macia M, Marin S, Bogusz-Czerniewicz M, Konstanty E, Modolel I, et al. Preoperative radiotherapy for rectal cancer: a comparative study of quality control adherence at two cancer hospitals in Spain and Poland. Radiol Oncol. 2014;48(2):210-8.

13. Torras MG, Canals E, Jurado-Bruggeman D, Marín-Borras S, Macià M, Jové J. Clinical audit of the radiotherapy process in rectal Cancer: clinical practice guidelines and quality certification do not avert variability in clinical practice. Transl Oncol. 2018;11(3):794-9.

14. Bujko K, Wyrwicz L, Rutkowski A, Malinowska M, Pietrzak L, Kryński J, et al. Long-course oxaliplatin-based preoperative chemoradiation versus $5 \times 5 \mathrm{~Gy}$ and consolidation chemotherapy for CT4 or fixed cT3 rectal cancer: results of a randomized phase III study. Ann Oncol. 2016;27(5):834-42.

15. MERCURY Study Group. Diagnostic accuracy of preoperative magnetic resonance imaging in predicting curative resection of rectal cancer: prospective observational study. BMJ. 2006;333(7572):779.

16. Teoh S, Muirhead R. Rectal radiotherapy--intensity-modulated radiotherapy delivery, delineation and doses. Clin Oncol (R Coll Radiol). 2016;28(2):93-102.

17. -Common Terminology Criteria for Adverse Events (CTAE). Version 4.0. Published: May 28, 2009 (v4.03: June 14, 2010). U. S. DEPARTMENT OF HEALTH AND HUMAN SERVICES (National Institutes of Health. National Cancer Institute.
18. -Taylor F, Mangat N, Swift IR, Brown G. Proforma-based reporting in rectal cancer. Cancer Imaging 2010;10 Spec no A:S142-50.

19. Siddiqui MRS, Shanmuganandan AP, Rasheed S, Tekkis P, Brown G, Abulafi AM. An audit comparing the reporting of staging MRI scans for rectal cancer with the London Cancer Alliance (LCA) guidelines. Eur J Surg Oncol. 2017:43(11):2093-104

20. Manchon-Walsh P, Aliste L, Espinàs JA, Prades J, Guarga A, Balart J, Biondo S, Castells A, Sanjuan X, Tabernero J, Borras JM, Catalonian rectal CancerGroup. Improving survival and local control in rectal cancer in Catalonia (Spain) in the context of centralisation: a full cycle audit assessment. Eur J Surg Oncol. 2016:42(12):1873-80.

21. van Leersum NJ, Snijders HS, Wouters MWJM, Henneman D, Marijnen CAM, Rutten HR, on behalf of the Dutch Surgical Colorectal Cancer Audit Group, et al. Evaluating national practice of preoperative radiotherapy for rectal cancer based on clinical auditing. Eur J Surg Oncol. 2013;39(9):1000-6.

22. -Wang SJ, Hathout L, Malhotra U, Maloney-Patel N, Kilic S, Poplin E, Jabbour SK. Decision-Making Strategy for Rectal Cancer Management Using Radiation Therapy for Elderly or Comorbid Patients. Int J Radiat Oncol Biol Phys 2018;15;100(4):926-944.

23. Montroni I, Ugolini G, Saur NM, Spinelli A, Rostoft S, Millan M, et al. Personalized management of elderly patients with rectal cancer: expert recommendations of the European Society of Surgical Oncology, European Society of Coloproctology, International Society of Geriatric Oncology, and American College of Surgeons Commission on Cancer. Eur J SurgOncol. 2018;44(11):1685-702.

24. Bese NS, Hendry J, Jeremic B. Effects of prolongation of overall treatment time due to unplanned interruptions during radiotherapy of different tumor sites and practical methods for compensation. Int J Radiat Oncol Biol Phys. 2007;68(3):654-61

25. van der Valk MJM, Hilling DE, Bastiaannet E, Meershoek-Klein Kranenbarg E, Beets GL, Figueiredo NL, Habr-Gama A. Et Al. long-term outcomes of clinical complete responders after neoadjuvant treatment for rectal cancer in the International Watch \& Wait Database (IWWD): an international multicentre registry study. Lancet. 2018;391(10139):2537-45.

26. Dossa F, Chesney TR, Acuna SA, Baxter NN, et al. A watch-and-wait approach for locally advanced rectal cancer after a clinical complete response following neoadjuvant chemoradiation: a systematic review and meta-analysis. Lancet Gastroenterol Hepatol. 2017;2(7):501-13.

27. Quirke P, Steele R, Monson J, Grieve R, Khanna S, Couture J, et al. Effect of the plane of surgery achieved on local recurrence in patients with operable rectal cancer: a prospective study using data from the MRC CR07 and NCICCTG CO16 randomised clinical trial. Lancet. 2009:373(9666):821-8.

28. Warrier SK, Kong JC, Guerra GR, Chittleborough TJ, Naik A, Ramsay RG, Lynch AC, Heriot AG. Risk factors associated with circumferential resection margin positivity in rectal Cancer: a Binational registry study. Dis Colon Rectum. 2018:61(4):433-40.

29. Whistance RN, Conroy T, Chie W, Costantini A, Sezer O, Koller M, et al. Clinical and psychometric validation of the EORTC QLQ-CR29 questionnaire module to assess health-related quality of life in patients with colorectal cancer. Eur J Cancer. 2009:45(17):3017-26.

\section{Publisher's Note}

Springer Nature remains neutral with regard to jurisdictional claims in published maps and institutional affiliations.

Ready to submit your research? Choose BMC and benefit from:

- fast, convenient online submission

- thorough peer review by experienced researchers in your field

- rapid publication on acceptance

- support for research data, including large and complex data types

- gold Open Access which fosters wider collaboration and increased citations

- maximum visibility for your research: over $100 \mathrm{M}$ website views per year

At $\mathrm{BMC}$, research is always in progress.

Learn more biomedcentral.com/submission 